\title{
Large Deviation Multifractal Analysis of a Class of Additive Processes with Correlated Non-Stationary Increments
}

\author{
Jacques Lévy Véhel \\ Regularity Team \\ INRIA Saclay-Ile-de-France, Orsay, France \\ e-mail: jacques.levy-vehel@inria.fr \\ Michał Rams \\ Institute of Mathematics, Polish Academy of Sciences \\ ul. Śniadeckich 8, 00-956 Warszawa, Poland \\ e-mail: rams@impan.gov.pl
}

\begin{abstract}
We consider a family of stochastic processes built from infinite sums of independent positive random functions on $\mathbb{R}_{+}$. Each of these functions increases linearly between two consecutive negative jumps, with the jump points following a Poisson point process on $\mathbb{R}_{+}$. The motivation for studying these processes stems from the fact that they constitute simplified models for TCP traffic. Such processes bear some analogy with Lévy processes, but are more complex since their increments are neither stationary nor independent. In [3], the Hausdorff multifractal spectrum of these processes were computed. We are interested here in their Large Deviation and Legendre multifractal spectra. These "statistical" spectra are seen to give, in this case, a richer information than the "geometrical" Hausdorff spectrum. In addition, our results provide a firm theoretical basis for the empirical discovery of the multifractal nature of TCP traffic.
\end{abstract}

Keywords. Multifractal processes, Hölder regularity, Large deviation multifractal spectrum, Legendre multifractal spectrum, Internet Traffic Control Protocol. AMS Classification. 28A80, 60G17, 60G30, 60J30.

\section{BACKGROUND AND MOTIVATIONS}

We study in this work a family of stochastic processes built from infinite sums of independent positive random functions on $\mathbb{R}_{+}$. Each of these functions increases linearly between two consecutive negative jumps, with the jump points following a Poisson point process on $\mathbb{R}_{+}$. The interest of this class of processes is twofold. The first is theoretical: they provide examples of additive processes with non-stationary and correlated increments which have a rich multifractal

supported by the EU FP6 Marie Curie programmes SPADE2 and CODY and by the Polish MNiSW Grant NN201 022233 'Chaos, fraktale i dynamika konforemna'. behaviour. More precisely, it was shown in [3] that their Hausdorff multifractal spectrum is non-trivial and is similar to the one of Lévy processes. We compute here their Large Deviation and Legendre multifractal spectra, and we show that they give an even more precise information than the Hausdorff spectrum.

The second interest stems from applications: the motivation for studying the processes considered here is that they constitute simplified but realistic models for TCP traffic on the Internet. Empirical studies, beginning with [19], [26], have shown that traffic on the Internet generated by the Traffic Control Protocol (TCP) is, under wide conditions, multifractal. This property has important consequences in practice. For instance, one may show that the queuing behavior of a multifractal traffic is significantly worse that the one of a non-fractal traffic (see [6] for details). It is therefore desirable to understand which features of TCP are responsible for multifractality, and maybe reduce their negative impact on, e.g., the queuing behavior.

"Explaining" the multifractality of traffic traces from basic features of the Internet is a difficult task. Models investigated so far have been based on the paradigm of multiplicative cascades ([6],[20]). Indeed, with few exceptions (notably [14], [16], [17]), multifractal analysis has mainly been applied to multiplicative processes. An obvious reason is that a multiplicative structure often leads naturally to multifractal properties ([22], [23]). However, there exists a number of real-world processes for which there is convincing experimental evidence of multifractality, but which do not display an associated multiplicative structure. Among these, a major example is Internet traffic: multiplicative models 
for TCP are not really convincing because there is no physical evidence that genuine traffic actually behaves as a cascading or multiplicative process. As a matter of fact, TCP traffic is rather an additive process, where the contributions of individual sources of traffic are merged in a controlled way.

The analysis developed below shows that merely adding sources managed by TCP does lead to a multifractal behavior. This result provides a theoretical confirmation to the empirical finding that TCP traffic is multifractal. Furthermore, it sheds light on the possible causes of this multifractality: indeed, it indicates that it may be explained from the very nature of the protocol, with no need to invoke a hypothetical multiplicative structure: it appears that multifractality in TCP arises from the interplay between the additive increase multiplicative decrease (AIMD) mechanism and the random non-synchronism of the sources. In addition, comparing the multifractal spectrum estimated numerically from TCP $\log$ s to our theoretical findings should allow to describe the fine structure of the hierarchy of sources ${ }^{1}$. Indeed, our computations permit to trace back, in a quantitative way, the main multifractal features of traces to specific mechanisms of TCP.

\section{A SIMPLIFIED MODEL OF TCP TRAFFIC}

The exact details of TCP seem too intricate to allow for a tractable mathematical analysis. We consider a simplified model that captures the main ingredients of the congestion avoidance and flow control mechanisms of TCP. For more details on TCP, one may consult [20], [28]. Our model goes as follows:

1) Each "source" of traffic $S_{i}$ sends "packets" of data at a time-varying rate. At time $t$, it sends $Z_{i}(t)$ packets.

2) Between two "consecutive" time instants $t$ and $t+d t$, two things may happen: the source $i$ may experience a "loss", i.e. the flow control mechanisms of TCP detects that a packet sent by the source did not reach its destination. In this case, TCP tries to avoid congestion by forcing the source to halve the number of packets sent at time $t+d t$ (multiplicative decrease mechanism). In other words, $Z_{i}(t+d t)=Z_{i}(t) / 2$. If there is no loss, the source is allowed to increase $Z_{i}(t)$ linearly, i.e. $Z_{i}(t+d t)=Z_{i}(t)+d t$ (additive increase mechanism).

3) The durations $\left(\tau_{k}^{(i)}\right)_{k \geq 1}$ between time instants $t_{k}$ and $t_{k+1}$ where a given source $i$ experiences a

\footnotetext{
${ }^{1}$ We note in passing that the spectra computed in this paper are the ones which are estimated from numerical data, in contrast to the Hausdorff spectrum obtained in [3], which is purely theoretical.
}

loss are modeled by a sequence of independent exponential random variables with parameter $\lambda_{i}$.

4) The total traffic $Z$ is the sum of an infinite number of independent sources with varying rates $\lambda_{i}$, where $\left(\lambda_{i}\right)_{i \geq 1}$ is a non-decreasing sequence of positive numbers.

As compared to the true mechanisms of TCP, our model contains a number of simplifications. However, except for one, these simplifications are not essential as far as multifractality is concerned. For instance, we believe that the fact that we ignore retransmission time out is of no consequence for our purpose: as will become clear below, imposing random silent periods with any sensible distribution for their length and occurrence should not change the pointwise regularity, as long as, at any time, there is an infinite number of active sources. Of all our assumptions, only the one of independence in 4) is clearly an oversimplification. Indeed, it is obvious that most losses are a consequence of congestion, which is caused by the fact that several sources are in competition. This gives rise to a strong correlation in the behavior of the sources. Introducing correlations would of course lead to a significantly more complex analysis. One should remark nevertheless that the competition between sources is implicitly taken into account in our model through the fact that sources indexed by large integers are subject to more frequent losses. Note also that most other approaches dealing with the fractal analysis of TCP make similar assumptions of independence: this is in particular the case for the popular "ON/OFF" models discussed below. In addition, we believe that incorporating some correlation in our model by letting the parameters $\lambda_{i}$ evolve in time and depend on the total traffic at each instant should be possible at the expense of some technicalities.

Our model takes into account the main features of TCP, while allowing at the same time a thorough mathematical analysis: we show in the sequel that $Z$ is multifractal, and we compute its Large Deviation and Legendre multifractal spectra. Both the multifractality of $Z$ and the shape of its spectra corroborates empirical findings [19], [26]. We remark here that the Large Deviation and Legendre multifractal spectra computed based on the increments of $Z$ differ from its Hausdorff multifractal spectrum obtained in [3]. As is shown below, the former give more information on $Z$ than the latter: more precisely, they reflect a fine property of the sequence $\left(\lambda_{i}\right)_{i \in \mathbb{N}}$ not detected by the Hausdorff spectrum. Another fact that makes the Large Deviation and Legendre multifractal spectra more relevant in applications is that these are the quantities actually 
estimated from numerical data, the Hausdorff spectrum being inaccessible from samples.

Let us briefly compare our approach with previous works dealing with the mathematical modeling of Internet traffic in relation with its (multi-) fractal behavior. A large number of studies [15], [18], [24] have given empirical evidence that many types of Internet traffic are "fractal", in the sense that they display self-similarity and/or long range dependence. Most theoretical models that have been developed so far have focused on explaining such behaviors. In that view, a popular class of models is based on the use of "ON/OFF" sources. An ON/OFF source is a source of traffic that is either idle, or sends data at a constant rate. Adequate assumptions on the distribution of the ON and/or OFF periods allow to obtain fractal properties. More precisely, the model in [18] considers independent and identically distributed ON/OFF sources, where the length of the ON and OFF periods are independent random variables. In addition, the distribution of the ON or/and of the OFF periods is assumed to have a regularly varying tail with exponent $\beta \in(1,2)$. Then, when the number of sources tends to infinity, and if one rescales time slowly enough, the resulting traffic, properly normalized, tends to a fractional Brownian motion, with exponent $3 / 2-\beta / 2$. In [25], it is shown that the same model leads to a $\beta$-stable Lévy motion when the time rescaling is "fast". The intermediate regime where time is rescaled proportionally to the number of sources is investigated in [13]. Another, elegant, model, which does not require a double re-normalization, is presented in [15]. It also uses a superposition of independent ON-OFF sources, but this time with a sequence of ratios for Poisson-idle and Poisson-active periods assumed to decay as a polynomial. Again, the resulting process display fractal features ${ }^{2}$.

A major feature of the above models is that the sources, in their ON mode, send data at a constant rate. This simplification does not take into account the strong and rapid variations induced by the flow control mechanisms of TCP. It seems to be of no consequence for studying long range dependence or self-similarity: these properties are obtained through the slow decay of the probability of observing large busy or idle periods. These slow decays may in turn be traced back to certain large scale features, such as, e.g., the distribution of the files sizes in the Internet [5]. More generally, it is usually accepted that long memory is a

\footnotetext{
${ }^{2}$ Note that the model that we consider does not require any kind of re-normalization.
}

property of the network. However, the use of ON/OFF sources does not allow a meaningful investigation of the multifractal properties of traffic: contrarily to long range dependence, multifractality is a short-time behavior. An ON/OFF modeling is clearly inadequate in this frame since it washes out all the (intra-source) high frequency content. At small time scales, the role of the protocol, i.e. TCP, becomes predominant [1]. Incorporating some sort of modeling of TCP is thus necessary if one wants to perform a sensible highfrequency analysis: the local fast variations due to TCP, are determinant from the multifractal point of view.

In that view, it is interesting to note that the limiting behavior of the ON/OFF model which is usually considered is the one leading to fractional Brownian motion. It is therefore not multifractal. In contrast, the other limiting case gives rise to a stable motion, which is multifractal. A possible cause might be that, in this regime, the inter-source high frequency content (i.e. the rapid variations in the total traffic resulting from de-synchronized sources) is large enough to produce multifractality. However, it is not clear which actual mechanisms in the Internet would favor this particular regime. It would also be interesting to investigate whether the critical case of [13] is also multifractal.

Another approach that allows to "explain" the multifractal features of TCP is based on the use of "fluid models" [1]: rather than representing TCP at the packet level, one uses fluid equations to describe the joint evolution of throughput for sessions sharing a given router. The interest of this approach is that it represents the traffic as simple products of random matrices, while allowing to capture the AIMD mechanism of TCP. In particular, [1] shows through numerical simulations that this model does lead to a multifractal behavior. In other words, the fluid model indicates that the multifractality is already a consequence of the AIMD mechanism. This numerical result corroborates our theoretical findings. A network extension of the fluid model is studied in [2]. It also points to multifractality of the traces, with additional intriguing fractal features.

\section{A CLASS OF ADDITIVE PROCESSES WITH} NON-STATIONARY AND CORRELATED INCREMENTS

We now describe our model in a formal way. Let $\left(\lambda_{i}\right)_{i \geq 1}$ be a non-decreasing sequence of positive numbers. These $\left(\lambda_{i}\right)_{i \geq 1}$ will describe the "mean activity" of individual sources of traffic.

For every $i \geq 1$, let $\left(\tau_{k}^{(i)}\right)_{k \geq 1}$ be a sequence of independent exponential random variables with parameter 
$\lambda_{i}$. Define $\tau_{0}^{(i)}=0$. Set

$$
T_{k}^{(i)}=\sum_{j=0}^{k} \tau_{j}^{(i)}
$$

The $\sigma$-algebras $\sigma\left(\tau_{k}^{(i)}, k \geq 1\right)$ are assumed to be mutually independent.

We consider an infinite sequence of sources $\left(S_{i}\right)_{i>1}$. The "traffic" $\left(Z_{i}(t)\right)_{t>0}$ generated by the source $S_{i}$, $i \geq 1$, is modeled by the following stochastic process

$Z_{i}(t)=\left\{\begin{array}{lc}Z_{i}(0)+t & \text { if } 0 \leq t<\tau_{1}^{(i)} \\ \frac{Z_{i}\left(T_{k-1}^{(i)}\right)+\tau_{k}^{(i)}}{\mu}+t-T_{k}^{(i)} & \text { if } T_{k}^{(i)} \leq t<T_{k+1}^{(i)}\end{array}\right.$

where $\left(Z_{i}(0)\right)_{i \geq 1}$ is a sequence of non-negative random variables such that the series $\sum_{i>1} Z_{i}(0)$ converge, and $\mu$ is a fixed real number larger than one (typically equal to 2 in the case of TCP).

The resulting "global traffic" is the process

$$
Z(t)=\sum_{i \geq 1} Z_{i}(t) \quad\left(t \in \mathbb{R}_{+}\right) .
$$

The following result is proved in [3]:

Proposition III.1. If $\sum_{i>1} 1 / \lambda_{i}<\infty$ then, with probability one, the stochastic process $Z$ is finite everywhere. If $\sum_{i \geq 1} 1 / \lambda_{i}=\infty$ then, with probability one, $Z(t)=\infty$ almost everywhere with respect to the Lebesgue measure.

Remark: the condition $\sum_{i \geq 1} 1 / \lambda_{i}<\infty$ may seem unnatural at first sight. However, it is easy to see that $\sum_{i \geq 1} 1 / \lambda_{i}=\infty$ implies a infinite mean rate of "traffic". As the second part of the Proposition shows, the resulting traffic is almost surely infinite in this case. The condition of finiteness also entails that most sources possess a high loss rate, and are thus "slow". Below, we will prove that this implies a multifractal behaviour. This is in line with the results of [27], where the authors show that multifractality may result from the existence of a few fast connections in a crowd of slow connections.

Note that each elementary process $Z_{i}$ may be decomposed in the following way on $\left[T_{k}^{(i)}, T_{k+1}^{(i)}\right)$ :

$$
Z_{i}=X_{i}+R_{i}
$$

with

$$
\left\{\begin{array}{l}
X_{i}(t)=t-T_{k}^{(i)} \\
R_{i}(t)=\frac{Z_{i}(0)}{\mu^{k}}+\frac{1}{\mu^{k+1}} \sum_{j=1}^{k} \mu^{j} \tau_{j}^{(i)} .
\end{array}\right.
$$

In [3], the multifractal nature of $Z$ was investigated through the computation of its Hausdorff multifractal spectrum. This spectrum gives a geometrical information on the singularity structure of $Z$. It was shown that the process $Z$, although it has correlated non-stationary increments, shares the Hausdorff multifractal spectrum of a Lévy process without Brownian part and whose characteristic measure is $\Pi=\sum_{i \geq 1} \lambda_{i} \delta_{-1 / \lambda_{i}}$.

In this work, we take another approach to multifractal analysis, based on a statistical description of the distribution of the singularities. It leads to the computation of the so-called Large Deviation multifractal spectrum and Legendre multifractal spectrum. These quantities are the ones usually considered in applications (see e.g. [26], [19], [20], [6]). We shall prove that $Z$ admits the same Large Deviation and Legendre multifractal spectra, which is however different from its Hausdorff spectrum and is described in Theorem III.4. We recall briefly the definitions of these spectra. Hausdorff multifractal spectrum

Let $X$ be a real valued function on a non-trivial subinterval $I$ of $\mathbb{R}$. The Hausdorff multifractal spectrum of $X$ describes, for every $\alpha \geq 0$, the "size" of the set $S_{\alpha}$ of points of $\operatorname{Int}(I)$ where $X$ has Hölder exponent $\alpha$. More precisely, the spectrum of singularities of $X$ is the function; $\alpha \mapsto f_{h}(\alpha)=\operatorname{dim}\left\{t: \alpha_{X}(t)=\right.$ $\alpha\}$, where dim denotes the Hausdorff dimension and $\alpha_{X}(t)$ the pointwise Hölder exponent of $X$ at $t$.

Large deviation multifractal spectrum

The Large Deviation spectrum $f_{g}(\alpha)$ is defined as:

$$
f_{g}(\alpha)=\lim _{\varepsilon \rightarrow 0} \limsup _{n \rightarrow \infty} \frac{\log N_{n}^{\varepsilon}(\alpha)}{\log n}
$$

where:

$$
N_{n}^{\varepsilon}(\alpha)=\#\left\{k: \alpha-\varepsilon \leq \alpha_{n}^{k} \leq \alpha+\varepsilon\right\}
$$

and $\alpha_{n}^{k}$ is the so-called "coarse grained" exponent corresponding to the interval $I_{n}^{k}=\left[\frac{k}{n}, \frac{k+1}{n}\right]$, i.e.:

$$
\alpha_{n}^{k}=\frac{\log \left|Y_{n}^{k}\right|}{-\log n}
$$

Here, $Y_{n}^{k}$ is a quantity that measures the variation of $X$ in $I_{n}^{k}$. The choice $Y_{n}^{k}=X\left(\frac{k+1}{n}\right)-X\left(\frac{k}{n}\right)$ leads to the simplest analytical computations. Taking $Y_{n}^{k}=\operatorname{osc}_{X}\left(I_{n}^{k}\right)$, i.e. the oscillation (that is, the supremum minus the infimum) of $X$ inside $I_{n}^{k}$, offers several theoretical and practical advantages. In this work, we shall however restrict to increments.

In the course of the proof, we will use intervals of the form $[s h,(s+1) h], s=0, \ldots, h^{-1}-1$ instead of the intervals $I_{n}^{k}=\left[\frac{k}{n}, \frac{k+1}{n}\right]$. There is no loss of generality in assuming that $h^{-1}$ is an integer. These intervals will be denoted $I_{h}^{s}$, and we define $N_{h}^{\varepsilon}(\alpha)$ accordingly. 
Legendre multifractal spectrum

It is natural to interpret the spectrum $f_{g}$ as a rate function in a large deviation principle (LDP). Large deviations theorems provide conditions under which such rate functions may be calculated as the Legendre transform of a limiting moment generating function. When applicable, this procedure provides a more robust estimation of $f_{g}$ than a direct computation.

Define, for $q \in \mathbb{R}$ :

$$
S_{n}(q)=\sum_{k=0}^{n-1}\left|Y_{n}^{k}\right|^{q}
$$

with the convention $0^{q}=0$ for all $q \in \mathbb{R}$. Let:

$$
\tau(q)=\liminf _{n \rightarrow \infty} \frac{\log S_{n}(q)}{-\log (n)} .
$$

The Legendre multifractal spectrum of $X$ is defined as the Legendre transform $\tau^{*}$ of $\tau$ :

$$
f_{l}(\alpha)=\tau^{*}(\alpha) \stackrel{\text { def }}{=} \inf _{q \in \mathbb{R}}(q \alpha-\tau(q)) .
$$

$f_{g}$ and $f_{l}$ are related as follows. Define the sequence of random variables $Z_{n}=\log \left|Y_{n}^{k}\right|$ where the randomness is through a choice of $k$ uniformly in $\{0, \ldots, n-1\}$. Consider the corresponding moment generating functions:

$$
c_{n}(q) \stackrel{\text { def }}{=}-\frac{\log \mathbb{E}_{n}\left[\exp \left(q Z_{n}\right)\right]}{\log (n)}
$$

where $\mathbb{E}_{n}$ denotes expectation with respect to $P_{n}$, the uniform distribution on $\{0, \ldots, n-1\}$. A version of Gärtner-Ellis Theorem [11] ensures that if $\lim c_{n}(q)$ exists (in which case it equals $1+\tau(q)$ ), and is differentiable, then $c^{*}=f_{g}-1$. In this case, one says that the weak multifractal formalism holds, i.e. $f_{g}=f_{l}$.

The relation $f_{g}^{* *}=f_{l}$ (which means that $f_{l}$ is the concave envelope of $f_{g}$ ) holds under rather weaker conditions. For instance, it is verified as soon as the support of $f_{g}$ is finite [21]. We shall use this result in the sequel to deduce $f_{l}$ from $f_{g}$.

Let us now return to our process. As we shall see, the three multifractal spectra of the sample paths of $Z$ (here $I=\mathbb{R}_{+}$) are governed by the following index

$$
\beta=\inf \left\{\gamma \geq 1 ; \sum_{i \geq 1} \frac{1}{\lambda_{i}^{\gamma-1}}<\infty\right\} .
$$

Note that $\beta \in[1,2]$ under the assumptions of Proposition III.1. More precisely, the result of [3] describing the Hausdorff multifractal spectrum of $Z$ is:
Theorem III.2. Assume $\sum_{i>1} 1 / \lambda_{i}<\infty$. With probability one, $Z$ is well defined and its Hausdorff multifractal spectrum is given by:

$$
f_{h}(\alpha)= \begin{cases}\beta \alpha & \text { if } \alpha \in[0,1 / \beta] \\ -\infty & \text { otherwise. }\end{cases}
$$

We will show that the Large Deviation and Legendre spectra depend additionally on the fine behaviour of the sequence $\left(\lambda_{i}\right)_{i \in \mathbb{N}}$ at infinity. This is in contrast with $f_{h}$ which depends only on $\beta$.

In that view, we shall need the following definitions. Fix $L>1$, and denote, for $k \in \mathbb{N}$ :

$$
L_{k}=L^{k}, M_{k}=\#\left\{\lambda_{j}<L_{k}\right\} .
$$

For $k \geq 2$, set $N_{k}=M_{k}-M_{k-1}$. Note that, by definition of $\beta$ :

$$
\forall \varepsilon_{0}>0, \exists K_{1}\left(\varepsilon_{0}\right): N_{k} \leq K_{1}\left(\varepsilon_{0}\right) L_{k}^{\beta-1+\varepsilon_{0}} .
$$

Likewise, there exists $K_{2}\left(\varepsilon_{0}\right)$ such that $M_{k} \leq$ $K_{2}\left(\varepsilon_{0}\right) L_{k}^{\beta-1+\varepsilon_{0}}$. In addition, for any given $\varepsilon_{0}>\overline{0}$, there exists a sequence $\left(a_{k}\right)_{k \in \mathbb{N}}$ increasing to infinity and such that:

$$
\forall k, N_{a_{k}} \geq L_{a_{k}}^{\beta-1-\varepsilon_{0}} .
$$

Definition III.3. The sequence $\left(\lambda_{i}\right)_{i \in \mathbb{N}}$ is said to be regular if, for any given $\varepsilon_{0}>0$, the sequence $\left(a_{k}-\right.$ $\left.a_{k-1}\right)_{k>1}$ is bounded.

When $\left(\lambda_{i}\right)_{i \in \mathbb{N}}$ is not regular, we set:

$$
\underline{\beta}=1+\liminf _{j \rightarrow \infty} \frac{\log \left(N_{j}\right)}{\log \left(L_{j}\right)},
$$

(so that $\left(\lambda_{i}\right)_{i \in \mathbb{N}}$ is regular if $\underline{\beta}=\beta$ ).

The main result of this work is:

Theorem III.4. Assume $\sum_{i>1} 1 / \lambda_{i}<\infty$ so that, with probability one, $Z$ is well defined.

1) Assume $\left(\lambda_{i}\right)_{i \in \mathbb{N}}$ is regular. Then, almost surely if $\beta<2$

$$
f_{g}(\alpha)= \begin{cases}\beta \alpha & \text { if } \alpha \in[0,1 / \beta] \\ 1+1 / \beta-\alpha & \text { if } \alpha \in[1 / \beta, 1+1 / \beta] \\ -\infty & \text { otherwise. }\end{cases}
$$

If $\beta=2$ then the same statement is true except that we only have the upper bound $f_{g}(\alpha)=$ $f_{l}(\alpha) \leq 3 / 2-\alpha$ for $\alpha \in(1 / 2,3 / 2]$.

2) Given any $1 \leq \beta_{1} \leq \beta_{2} \leq 2$, there exits a sequence $\left(\lambda_{i}\right)_{i \in \mathbb{N}}$ such that $\underline{\beta}=\beta_{1}, \beta=\beta_{2}$, 
and, almost surely:

$$
f_{g}(\alpha)= \begin{cases}\beta_{2} \alpha & \text { if } \alpha \in\left[0,1 / \beta_{2}\right] \\ 1 & \text { if } \alpha \in\left[1 / \beta_{2}, 1 / \beta_{1}\right] \\ 1+1 / \beta_{1}-\alpha & \text { if } \alpha \in\left[1 / \beta_{1}, 1+1 / \beta_{1}\right. \\ -\infty & \text { otherwise. }\end{cases}
$$

In all cases, the equality $f_{g}(\alpha)=f_{l}(\alpha)$ holds.

As can be seen from this result, the Large Deviation and Legendre spectra display a decreasing part for "large" $\alpha$ not present in the Hausdorff spectrum. This comes from the fact that we are using increments to define the spectra, and is similar to what occurs for instance for fractional Brownian motion (see [14]). We conjecture that, as is the case for fractional Brownian motion, the decreasing part is not present if oscillations are used in place of increments.

In addition, $f_{g}$ and $f_{l}$ give more information on the structure of "traffic" than $f_{h}$, since they detect a fine feature of the sequence $\left(\lambda_{j}\right)_{j}$, i.e. the case where $\beta \neq$ $\beta$, to which $f_{h}$ is insensitive. This is another instance where the statistical spectra are more relevant both in theory and in applications that the geometrical one.

\section{PRACTICAL CONSEQUENCES FOR TCP TRAFFIC}

According to our analysis, and as the proof of Theorem III.4 will show, multifractality is the consequence of the many discontinuities of all sizes entailed by the multiplicative decrease mechanism or retransmission time out. This is in agreement with experimental evidence as reported for instance in [7]. Simulations performed there indicate that retransmission time out plays a crucial role for the multifractal nature of TCP.

It is a well documented fact multifractality has a significant and negative impact on performance (see, e.g. [12], [6]). As compared for instance to a monofractal traffic, a multifractal one results in much larger average queue lengths, specially at lower utilizations.

This effect has been quantified in various ways. We consider as an example the approaches proposed in [12] and [6]. Both references remark that the scaling behaviour alone (i.e. the spectrum, or more precisely its Legendre transform) is not sufficient to assess the queueing behaviour, and that the prefactors ruling the magnitude of the fluctuations are needed. In [12], these are obtained from a "boundary condition", while they are explicitly needed in [6]. Using formulas (5), (6) and (7) of [12], we estimated the maximum number of sources $N$ that can be supported for given parameters of the network (we chose the same paremeters as in [12]) in our model with $\beta=1$ and $\beta=2$. For a link speed of $1.54 \mathrm{Mb} / \mathrm{s}$, [12] gives $N=0.34$ for an fBm modeling and $N=0.23$ for a model based on a multiplicative cascade. Our model yields $N=0.18(\beta=2)$ and $N=0.16(\beta=1)$. For a ];ink speed of $2.05 \mathrm{Mb} / \mathrm{s}$, the values are $0.4(\mathrm{fBm})$, 0.27 (cascade), $0.22(\beta=2)$ and $0.19(\beta=1)$. Finally, for a link speed of $10 \mathrm{Mb} / \mathrm{s}$, the values are 0.68 (fBm), 0.64 (cascade), $0.54(\beta=2)$ and $0.5(\beta=1)$. One sees that in our model the predictions are even worse than with a multiplicative cascade. In addition, in agreement with previous studies, we find that "more multifractality", i.e. a wider spectrum, degrades the performances: indeed, the width of the spectrum in our model is $1+\frac{1}{\beta}=3 / 2$ if $\beta=2$ and 2 if $\beta=1$ (for simplicity, we consider only the regular case).

Let us now turn to the approach in [6]. Formula (5) in this article gives an explicit expression for the logarithm of the probability for the queue tail asymptotic, provided both the spectrum and the prefactor are known. Since we did not compute the prefactor, we cannot use directly Formula (5). However, making the reasonable assumption that these do not depend on $\beta^{3}$, we are able to compare the asymptotic probabilities for different values of $\beta$ : plotting the part of the right hand side of Formula (5) that depends on the spectrum shows that the minimum giving the value of the probability is a decreasing function of $\beta$. As a consequence, a narrow spectrum (a large $\beta$ ) yields better performances than a wide one, with small $\beta$.

The question is then to find possible ways to reduce multifractality. Since in our model multifractality is due the discontinuities caused by retransmission time out, one may wonder whether a different decrease policy would enhance the situation. The answer is in the negative, as shown by the next theorem, which is natural extension of Theorem III.4. Let $\left(\mu_{i}\right)_{i \geq 1} \in$ $(1, \infty)^{\mathbb{N}^{*}}$. For every $i \geq 1$ define

$$
\widetilde{Z}_{i}(t)=\left\{\begin{array}{lc}
Z_{i}(0)+t & \text { if } 0 \leq t<\tau_{1}^{(i)} \\
\frac{\widetilde{Z}_{i}\left(T_{k-1}^{(i)}\right)+\tau_{k}^{(i)}}{\mu_{i}}+t-T_{k}^{(i)} & \text { if } T_{k}^{(i)} \leq t<T_{k+1}^{(i)}
\end{array}\right.
$$

and

$$
\widetilde{Z}(t)=\sum_{i \geq 1} \widetilde{Z}_{i}(t)
$$

Theorem IV.1. Assume $\left(\mu_{i}\right)_{i \geq 1}$ is bounded, $\mid \log \left(\mu_{i}-\right.$ $1) \mid=o\left(\log \left(\lambda_{i}\right)\right)$ and $\sum_{i \geq 1} 1 /\left(\mu_{i}-1\right) \lambda_{i}<\infty$. With probability one, the conclusions of theorem III.4 hold.

In other words, the multifractal nature of $Z$ is not affected if $\mu$ is replaced by $\mu_{i}$ in $Z_{i}$ and if the sequence $\left(\mu_{i}\right)$ remains bounded and does not tend "too fast"

\footnotetext{
${ }^{3}$ in the case of $\mathrm{fBm},[6]$ shows that the prefactor do not depend on the value of $H$.
} 
to 1 . Theorem IV.1 includes many potential or actual variants of TCP. For instance, one could imagine treating in different ways sources with different intensity $\lambda_{i}$ : as long as the reduction factors are bounded and do not approach 1 too fast, the multifractal spectrum remains unchanged. This suggests that reducing the multifractality of TCP cannot be achieved in this way.

Instead of changing the size of the jumps, another possibility would be to reduce the frequency of their occurrence. It is well-known that avoiding retransmission time out improves the performances. One way to do so is to use fast recovery. Experimental results in [7] show that this mechanism reduces multifractality in the sense of narrowing the multifractal spectrum. [8] studies the influence of the RED mechanism, which also allows to lower the occurrence of retransmission time out. It shows that RED both improves throughput and delay performances and reduces multifractality in the same sense. Again, this is in agreement with our analysis, since avoiding retransmission time out reduces the number of jumps: let us explain on a simple example why this indeed decreases multifractality in our model. Suppose that $\lambda_{i}=i^{\delta}$ for some $\delta>1$. It is easily checked that this yields $\beta=1+\frac{1}{\delta}$. The width of the spectrum is thus $1+\frac{1}{\beta}=1+\frac{\delta}{\delta+1}$, which is an increasing function of $\delta$ : a narrow spectrum translates into a small $\delta$. Since the average time interval between two jumps is $i^{-\delta}$, we see that reducing multifractality amounts to decreasing the number of retransmission time out for each source.

The remaining of this work is devoted to the proof of Theorem III.4.

\section{SOME ANCILLARY RESUlts}

We gather in this section some properties of the processes $Z_{m}$ and their increments that will be needed in the sequel.

Although each process $Z_{m}$ is not stationary, it possesses a stationary distribution, and moreover, convergence to this stationary distribution occurs exponentially fast. To make this precise, we first remark that for each source $m, Z_{m}$ belongs to the class of processes known as piecewise deterministic Markov processes. See [9] for a thorough account on this topic. For such processes, [4] has studied conditions ensuring ergodicity, i.e. the existence of an invariant measure $G_{m}$ such that, for all $x$ :

$$
\lim _{t \rightarrow \infty}\left\|P_{m}^{t}(x, .)-G_{m}(.)\right\|=0,
$$

where $\|$.$\| denotes the total variation norm and$ $P_{m}^{t}(x, A)=P_{x}\left(Z_{m}(t) \in A\right)\left(P_{x}\right.$ is the probability when the process starts from $x$ ). It is straightforward to check that Theorem 5.4 in [4] applies to $Z_{m}$, so that each source is indeed ergodic. In the proofs below, we will however need a bit more than ergodicity: we will require that convergence in (5) takes place at an exponential rate. To check this, we make use of powerful results proved in [10] for general Markov processes. Again, it is easy to verify that Theorem 7.1 of [10] applies, to the effect that:

Proposition V.1. For all $t \geq 0$ :

$$
\left\|P_{m}^{t}(x, .)-G_{m}(.)\right\| \leq M(x) \rho^{t},
$$

for some finite $M(x)$ and where $\rho<1$.

In particular, the above proposition means that, for a source with intensity $\lambda$ starting at time $t$ from an arbitrary state, we are arbitrarily close to the stationary state at time $t+\lambda T$ when $T>>1$. This fact will be used in the sequel.

The stationary distribution $G_{m}$ is absolutely continuous and we now state some of the properties of its density $g_{m}$. We will first need a bound from below on the tail of the density probability $g_{m}$ :

Lemma V.2. For all $x$ and all $d \geq 0$,

$$
g_{m}(x+d) \geq e^{-\lambda_{m} d} g_{m}(x) .
$$

Proof: Note first that, by rescaling, we get that $g(x) \stackrel{\text { def }}{=} \frac{1}{\lambda_{m}} g_{m}\left(\frac{x}{\lambda_{m}}\right)$ does not depend on $m$. So we may take without loss of generality $\lambda_{m}=1$ in the proof. If the process at some time $t$ is between $x$ and $x+\varepsilon$, after a duration $d$, if there are no jumps (which occurs with probability $e^{-d}$ ), its value will be between $x+d$ and $x+d+\varepsilon$. As we are dealing with the stationary distribution, we get

$$
\int_{x+d}^{x+d+\varepsilon} g(s) d s \geq e^{-d} \int_{x}^{x+\varepsilon} g(s) d s .
$$

We now give a bound from below on the density probability $g_{m}$ close to the origin:

Lemma V.3. for all $x \leq 1 / \lambda_{m}$,

$$
g_{m}(x) \geq c \lambda_{m} e^{-c\left(\log \left(\lambda_{m} x\right)\right)^{2}}
$$

for a fixed constant $c>0$.

Proof: Again, we assume $\lambda_{m}=1$. When the process is in the stationary state, the following relation holds for all times $t$ and all $\varepsilon>0$ :

$P\left(Z_{m}(t)<x, Z_{m}(t+\varepsilon)>x\right)=P\left(Z_{m}(t)>x, Z_{m}(t+\varepsilon)<x\right)$. 
We re-write this inequality in terms of density:

$e^{-\varepsilon} \int_{x-\varepsilon}^{x} g(s) d s=\left(1-e^{-\varepsilon}\right) \int_{x}^{\mu(x-\varepsilon)} g(s) d s+O\left(\varepsilon^{2}\right)$.

Letting $\varepsilon$ tend to 0 , we get:

$$
g(x)=\int_{x}^{\mu x} g(s) d s .
$$

As a consequence,

$$
\begin{aligned}
g(x) & \geq \int_{\sqrt{\mu} x}^{\mu x} g(s) d s \\
& \geq(\mu-\sqrt{\mu}) x g(y(x)),
\end{aligned}
$$

where $y(x) \in[x \sqrt{\mu}, x \mu]$. Denote $\left.y^{(1)}(x)=y(x)\right)$ and $y^{(n)}(x)=y\left(y^{(n-1)}(x)\right)$. Now let $n(x)$ be the smallest $n$ for which $y^{(n)}(x) \geq 1$ (note that $y^{(n)}(x) \leq \mu$ ). Now:

$$
\frac{-\log (x)}{\log (\mu)} \leq n(x) \leq \frac{-\log (x)}{\log (\sqrt{\mu})} \text {. }
$$

Thus:

$$
g(x) \geq(\mu-\sqrt{\mu})^{n(x)} g\left(y^{(n(x))}\right) \prod_{k=0}^{n(x)-1} y^{(k)}(x) .
$$

We obtain a lower bound for each of the three terms in the product on the right hand side of the above inequality. First,

$$
g\left(y^{(n(x))}\right) \geq \inf _{s \in[1, \mu]} g(s) \geq e^{1-\mu} g(1)=\text { constant } .
$$

Then,

$$
(\mu-\sqrt{\mu})^{n(x)} \sim e^{-c_{1} \log x},
$$

where $c_{1}$ is a positive constant. Finally,

$$
\prod_{k=0}^{n(x)-1} y^{(k)}(x) \geq \prod_{k=0}^{-\log (x) / \log (\sqrt{\mu})} \mu^{-k / 2} \sim e^{-c_{2}(\log x)^{2}},
$$

where $c_{2}$ is again a positive constant.

Now we will consider the stationary distribution of the increments of source $m$ within some time interval $[t, t+h]$, where $0<h<1$. We denote by $\Delta Z_{m}$ these increments, i.e. $\Delta Z_{m}=Z_{m}(t+h)-Z_{m}(t)$. The probability distribution of this random variable has an atom at $h$ with size $e^{-\lambda_{m} h}$. The rest of this distribution is absolutely continuous, and we denote density of this absolute continuous part by $z_{m}$.

First, we give a bound for the variance of $\Delta Z_{m}$ :

\section{Lemma V.4.}

$$
\operatorname{var} \Delta Z_{m} \leq K_{0} \min \left(\frac{h}{\lambda_{m}}, \frac{1}{\lambda_{m}^{2}}\right) \text {. }
$$

Proof: This results from Lemma 9 and 10 in [3].
The following bound on $z_{m}$ will be useful:

Lemma V.5. For all $x$ and for all $d \geq 0$

$$
z_{m}(x-d) \geq \exp \left(-\frac{\lambda_{m} \mu d}{\mu-1}\right) z_{m}(x) \text {. }
$$

Proof: Here again, we may reason in the case $\lambda=1$ and denote $Z_{1}$ the corresponding source. The value of $\Delta Z_{1}$ is uniquely determined by the value of $Z_{1}$ at time $t$ plus the times of the jumps in the interval $[t, t+h]$. Since almost surely, there will be a finite number of jumps in $[t, t+h]$, we may decompose $\Delta Z_{1}$ as $\Delta Z_{1}=\sum_{k=0}^{\infty} \Delta Z_{1}^{(k)}$, where $\Delta Z_{1}^{(k)}=$ $\Delta Z_{1}^{(k)}\left(Z(t) ; t_{1}, \ldots t_{k}\right)$ is the increment when there are precisely $k$ jumps (occurring at times $t_{1}, \ldots t_{k}$ ). Note that all the $\Delta Z_{1}^{(k)}$ are mutually exclusive events. In this lemma, we are interested in the absolute continuous part of $\Delta Z_{1}$, so we assume that there is at least one jump in $[t, t+h]$, i.e. we exclude the case $k=0$. Then $z_{1}=\sum_{k=1}^{\infty} z_{1}^{(k)}$, where $z_{1}^{(k)}$ denotes the density of $\Delta Z_{1}^{(k)}$. In view of (1), we have:

$\Delta Z_{1}^{(k)}\left(x+s ; t_{1}, \ldots t_{k}\right)=\Delta Z_{1}^{(k)}\left(x ; t_{1}, \ldots t_{k}\right)+s\left(\frac{1}{\mu^{k}}-1\right)$.

The distribution of the times $t_{1}, \ldots, t_{k}$ is independent of $Z_{1}(t)$, hence applying lemma V.2, we get:

$$
z_{1}^{(k)}(x-d) \geq e^{-d \frac{\mu^{k}}{\mu^{k}-1}} z_{1}^{(k)}(x) .
$$

We conclude by using that $\frac{\mu^{k}}{\mu^{k}-1} \leq \frac{\mu}{\mu-1}$.

This lemma entails the following corollary:

Corollary V.6. For $a<b \leq c<d$,

$\int_{c}^{d} z_{m}(x) d x \leq \frac{d-c}{b-a} \exp \left(\lambda_{m}(d-a) \frac{\mu}{\mu-1}\right) \int_{a}^{b} z_{m}(x) d x$.

\section{Proof OF TheOREM III.4}

We will prove that the Large Deviation spectrum $f_{g}$ indeed verifies (3) and (4) as described in Theorem III.4. The results for the Legendre spectrum $f_{l}$ immediately follow by noting that $f_{g}$ has finite support and is concave, thus, by [21], $f_{l}=f_{g}^{* *}=f_{g}$.

Note first the obvious but useful fact below, that we are going to employ throughout the proof. We will use the following notation: if $A$ is an event or random variable that depends only on the values of $Z$ and $Z_{i}$ over the interval $I_{h}^{0}$ of length $h$, we will write $A(s)$ for the event or random variable obtained through replacing $I_{h}^{0}$ by $I_{h}^{s}$. Thus, in particular, $A(0)=A$.

Lemma VI.1. Assume $P(A(s)) \cdot E(B(s) \mid A(s)) \leq P$. Then for all $N>0$

$$
P\left(\sum_{s ; A(s)} B(s) \geq N P h^{-1}\right) \leq N^{-1}
$$


Proof:

$$
E \sum_{s ; A(s)} B(s)=\sum_{s} P(A(s)) E(B(s) \mid A(s)) \leq P h^{-1} .
$$

Except in section VI-B2 where we compute a lower bound for the case $\alpha \geq 1 / \beta$, we will use this lemma with $B \equiv 1$, i.e. we estimate the number of $s$ for which $A(s)$ happens. This will let us obtain upper bounds for the spectrum based only on estimating the distribution of $\alpha_{h}^{s}$ under assumption that the initial state of the process $Z$ is the stationary distribution (see section VI-A1 for a more precise statement). Obviously, the lower bounds are unattainable in such a simple way we will have to take into account correlations between $\alpha_{h}^{s}$ for different $s$.

Our goal is to give almost sure estimations of $N_{h}^{\varepsilon}$. In the course of the proof, we are going to assume certain events to happen almost surely, even though their probability is, for each fixed $h$, smaller than 1 . We can choose a subsequence $\left\{h_{i}\right\}$ for which the series of probabilities that those events do not hold is summable. Routine application of the Borel-Cantelli Lemma ensures that, almost surely, there exists $N_{0}$ such that those events are all true for all $\left\{h_{i} ; i>N_{0}\right\}$. Moreover, except in the case of the lower bound estimates for $\alpha \geq 1 / \beta$, the probabilities of those events will be at least $1-h^{c}$, hence (by Borel-Cantelli Lemma again), those events are almost surely true for almost all elements in any exponentially decreasing sequence $\left\{h_{i}\right\}$. We skip these technical but elementary details in order to make the proofs more legible.

We shall use the following notations:

$$
T_{l}=\sum_{\lambda_{j}>l} \Delta Z_{j}, T_{l}^{+}=\sum_{\lambda_{j} \leq l} \Delta Z_{j}, Y_{m}=\sum_{j \neq m} \Delta Z_{j} .
$$

\section{A. Case $\alpha<1 / \beta$}

1) Upper bound: Choose $\varepsilon_{0}<(1-\alpha \beta) / 2$. We will assume $Z$ is in stationary state at time $t$, hence it stays in this state ever after. We start by estimating the variance of the tail on an arbitrary interval $I_{h}^{s}$ :

$$
\operatorname{var} T_{h^{-\alpha}} \leq \sum_{L_{k}>h^{-\alpha}} N_{k} \max _{\lambda_{j}>L_{k-1}} \operatorname{var} \Delta Z_{j} \leq S_{1}+S_{2}
$$

where

$$
S_{1}=\sum_{L_{k}=h^{-\alpha}}^{h^{-1}} K_{0} K_{1}\left(\varepsilon_{0}\right) L h L_{k}^{\beta-2+\varepsilon_{0}}
$$

and

$$
S_{2}=\sum_{L_{k}=h^{-1}}^{\infty} K_{0} K_{1}\left(\varepsilon_{0}\right) L^{2} L_{k}^{\beta-3+\varepsilon_{0}} .
$$

Both those series are exponential, hence may be estimated by a constant times the maximal summand. For $\beta=2$ we have

$$
S_{1} \approx c\left(\varepsilon_{0}\right) h^{1-\varepsilon_{0}}
$$

(here and below, $f(h) \approx g(h)$ means that there exist two positive finite constants $a, b$ such that, for all $h<$ 1 , af $(h) \leq g(h) \leq b f(h))$. For $\beta<2$ :

$$
S_{1} \approx c\left(\varepsilon_{0}\right) h^{1+\left(2-\beta+\varepsilon_{0}\right) \alpha} .
$$

At the same time,

$$
S_{2} \approx c\left(\varepsilon_{0}\right) h^{3-\beta-\varepsilon_{0}}
$$

which is of at most the same order as $S_{1}$. Let $A_{1}$ be the event that $\left|T_{h^{-\alpha}}\right|<h^{\alpha} / 2$. By Chebyshev inequality,

$$
P\left(A_{1}\right) \geq 1-c\left(\varepsilon_{0}\right) h^{1-\alpha \beta-\varepsilon_{0}} .
$$

We have:

$$
\begin{aligned}
P\left(|\Delta Z| \geq h^{\alpha}\right) & \leq 1-P\left(A_{1}\right)+P\left(|\Delta Z| \geq h^{\alpha} \mid A_{1}\right) \\
& \leq 1-P\left(A_{1}\right)+P\left(\left|T_{h^{-\alpha}}^{+}\right| \geq \frac{1}{2} h^{\alpha}\right)
\end{aligned}
$$

If for all $\lambda_{j} \leq h^{-\alpha}$ we had $\Delta Z_{j}=h$ then

$$
\begin{aligned}
T_{h^{-\alpha}}^{+} & \leq h K_{2}\left(\varepsilon_{0}\right) h^{-\alpha\left(\beta-1+\varepsilon_{0}\right)} \\
& =K_{2}\left(\varepsilon_{0}\right) h^{\alpha} h^{1-\alpha \beta-\alpha \varepsilon_{0}} \\
& <\frac{1}{2} h^{\alpha}
\end{aligned}
$$

for $h$ small enough. Hence,

$$
\begin{aligned}
P\left(\left|T_{h^{-\alpha}}^{+}\right| \geq \frac{1}{2} h^{\alpha}\right) & \leq P\left(\exists \lambda_{j} \leq h^{-\alpha} ; \Delta Z_{j}<h\right) \\
& \leq E \sharp\left\{\lambda_{j} \leq h^{-\alpha} ; \Delta Z_{j}<h\right\} \\
& =\sum_{\lambda_{j} \leq h^{-\alpha}}\left(1-e^{-h \lambda_{j}}\right) \\
& \leq \sum_{L_{k} \leq h^{-\alpha}} N_{k}\left(1-e^{-h L^{-1} L_{k}}\right) \\
& \leq \sum_{L_{k} \leq h^{-\alpha}} K_{1}\left(\varepsilon_{0}\right) L_{k}^{\beta-1+\varepsilon_{0}} h L^{-1} L_{k} \\
& \approx c\left(\varepsilon_{0}\right) h^{1-\alpha \beta-\alpha \varepsilon_{0}} .
\end{aligned}
$$

Substituting into (8) and applying (7) we obtain

$$
P\left(|\Delta Z|>h^{\alpha}\right) \leq c\left(\varepsilon_{0}\right) h^{1-\alpha \beta-\varepsilon_{0}} .
$$

We now apply Lemma VI.1 with $A=\left\{|\Delta Z|>h^{\alpha}\right\}$, $B=1$ and $N=h^{-\varepsilon_{0}}$. This yields that, with probability at least $1-h^{\varepsilon_{0}}, A(s)$ is satisfied for at most 
$c\left(\varepsilon_{0}\right) h^{-\alpha \beta-2 \varepsilon_{0}}$ intervals $I_{h}^{s}$. For those $h$ for which this is true, we obtain the estimation

$$
N_{h}^{\varepsilon}(\alpha) \leq(\alpha-\varepsilon) \beta+3 \varepsilon_{0}
$$

where $\varepsilon_{0}$ may be chosen arbitrarily small. Hence:

$$
f_{g}(\alpha) \leq \alpha \beta
$$

2) Lower bound: Choose $\varepsilon_{0}$ as in the previous subsection and a large enough constant $M$ (to be determined later).

Let $h$ and $a_{k}$ be such that $L_{a_{k}} \in$ $\left(h^{-\alpha+\varepsilon_{0}}, M h^{-\alpha+\varepsilon_{0}}\right)$. Note that, in the regular case, for every $h$, one may find $a_{k}$ that will satisfy this assumption (provided $M$ is chosen large enough).

Applying Lemma VI.1 to event $A_{1}$ with $N=h^{-\varepsilon_{0}}$ and $B=1$ we see that, with probability $1-h^{\varepsilon_{0}}$, $A_{1}(s)$ is satisfied for all except at most $c\left(\varepsilon_{0}\right) h^{-\alpha \beta-2 \varepsilon_{0}}$ intervals $I_{h}^{s}$.

For all $\lambda_{j} \in\left(L^{-1} L_{a_{k}}, L_{a_{k}}\right)$, we denote by $A_{2}^{j}$ the event $\left\{\Delta Z_{j}<-2 h^{\alpha}\right\}$. Let $A_{2}=\bigcup A_{2}^{j}$. If both $A_{1}(s)$ and $A_{2}^{m}(s)$ (for some $m$ ) are satisfied then

$$
\begin{aligned}
\Delta Z(s) & \leq-2 h^{\alpha}+\frac{1}{2} h^{\alpha}+\sum_{\lambda_{j} \leq h^{-\alpha} ; j \neq m} h \\
& \leq-\frac{3}{2} h^{\alpha}+h K_{2}\left(\varepsilon_{0}\right) h^{-\alpha\left(\beta-1+\varepsilon_{0}\right)} \\
& \leq-h^{\alpha}
\end{aligned}
$$

for $h$ small enough. Hence, $A_{1}(s) \cap A_{2}(s)$ is a sufficient condition for $|\Delta Z| \geq h^{\alpha}$.

For the event $A_{2}^{j}(s)$ to happen, it is enough that $Z_{j}$ has a jump inside $I_{h}^{s}$ and that it had previously no jump for a duration at least equal to $\frac{2 \mu}{\mu-1} h^{\alpha}$ (recall that $\left.h<<h^{\alpha}\right)$. As $\lambda_{j} \leq M h^{-\alpha+\varepsilon_{0}}<<h^{-\alpha}$, this happens a number of times of the order of $h^{-\alpha+\varepsilon_{0}}$ for most $j$.

All events $A_{2}^{j}$ are independent (since the processes $Z_{j}$ are independent). Hence, though it is possible that $A_{2}^{j_{1}}(s)$ and $A_{2}^{j_{2}}(s)$ both happen for the same $s$, the number of such situations is (with arbitrarily large probability if $h$ is small enough) negligible. As $j$ may take at least $h^{\left(-\alpha+\varepsilon_{0}\right)\left(\beta-1-\varepsilon_{0}\right)}$ different values, $A_{2}(s)$ is thus satisfied for at least $2 h^{-\alpha \beta+\varepsilon_{0}(\alpha+\beta)}$ different values of $s$.

Events $A_{1}$ and $A_{2}$ are independent because the former depends only on the tail $\left(Z_{j}\right.$ with $\left.\lambda_{j}>h^{-\alpha}\right)$ while the latter depends only on some $Z_{j}$ with $\lambda_{j} \approx$ $h^{-\alpha+\varepsilon_{0}}$. Hence, with arbitrarily large probability (for $h$ small enough) $\left(A_{1} \cap A_{2}\right)(s)$ is satisfied for at least $h^{-\alpha \beta+\varepsilon_{0}(\alpha+\beta)}$ different values of $s$.

We have thus proved that $|\Delta Z| \geq h^{\alpha}$ for at least $h^{-\alpha \beta+\varepsilon_{0}(\alpha+\beta)}$ intervals $I_{h}^{s}$ if $h$ is small enough. In the previous subsection, we have shown that $|\Delta Z| \geq h^{\alpha-\varepsilon}$ for at most $h^{-(\alpha-\varepsilon) \beta-3 \varepsilon_{0}}$ intervals $I_{h}^{s}$. Thus,

$$
N_{h_{i}}^{\varepsilon}(\alpha) \geq \alpha \beta-\varepsilon_{0}(\alpha+\beta)
$$

provided $\beta \varepsilon>(3+\alpha+\beta) \varepsilon_{0}$. Choosing for all $\varepsilon$ a corresponding $\varepsilon_{0}$ and sufficiently small $h$ for (11) to hold and then passing to the limit $\varepsilon \rightarrow 0$, we get

$$
f_{g}(\alpha) \geq \alpha \beta
$$

\section{B. Case $\alpha \geq 1 / \beta$}

1) Upper bound: There is no need to give an upper bound for $\alpha=1 / \beta$, so we will assume $\alpha>1 / \beta$ in this subsection. Choose $\varepsilon_{0}$ sufficiently small so that $\alpha \geq 1 / \beta+\varepsilon_{0}+3 \varepsilon$. Choose a large enough constant $M$. Assume that $Z$ is in its stationary state at time $t$.

Let $h$ and $a_{k}$ be such that $L_{a_{k}} \in$ $\left(h^{-1 / \beta-\varepsilon_{0}}, M h^{-1 / \beta-\varepsilon_{0}}\right)$. Like in the previous subsection, in the regular case one can find, for every $h, a_{k}$ that will satisfy this assumption (provided $M$ was chosen big enough).

We denote by $A_{3}$ the event that there exists $\lambda_{m} \in$ $\left(L^{-1} L_{a_{k}}, L_{a_{k}}\right)$ such that $\Delta Z_{m}<h$. If $\beta=1$ then

$$
P\left(A_{3}\right) \geq 1-e^{-h L_{a_{k}}}=1-e^{-h^{-\varepsilon_{0}}}
$$

otherwise we know that there are at least $L_{a_{k}}^{\beta-1-\varepsilon_{0}}$ different $\lambda_{m} \in\left(L^{-1} L_{a_{k}}, L_{a_{k}}\right)$, hence

$$
\begin{aligned}
P\left(A_{3}\right) & \geq 1-\left(e^{-h L^{-1} L_{a_{k}}}\right)^{L_{a_{k}}^{\beta-1-\varepsilon_{0}}} \\
& \geq 1-e^{-L^{-1} h^{-\varepsilon_{0}\left(\beta-1 / \beta-\varepsilon_{0}\right)}}
\end{aligned}
$$

for $\varepsilon_{0}<\beta-1$. We may write

$$
\begin{gathered}
P\left(\Delta Z \in\left(h^{\alpha+\varepsilon}, h^{\alpha-\varepsilon}\right) \cup\left(-h^{\alpha-\varepsilon},-h^{\alpha+\varepsilon}\right)\right) \leq \\
1-P\left(A_{3}\right)+P\left(\Delta Z \in\left(h^{\alpha+\varepsilon}, h^{\alpha-\varepsilon}\right) \cup\left(-h^{\alpha-\varepsilon},-h^{\alpha+\varepsilon}\right) \mid A_{3}\right) .
\end{gathered}
$$

As $\Delta Z=\Delta Z_{m}+Y_{m}$, we have

$$
\begin{aligned}
& P\left(\Delta Z \in\left(h^{\alpha+\varepsilon}, h^{\alpha-\varepsilon}\right) \cup\left(-h^{\alpha-\varepsilon},-h^{\alpha+\varepsilon}\right) \mid A_{3}\right)= \\
& \int d Y_{m}(a)\left(\int_{h^{\alpha+\varepsilon}-a}^{h^{\alpha-\varepsilon}-a}+\int_{-h^{\alpha-\varepsilon}-a}^{-h^{\alpha+\varepsilon}-a}\right) z(x) d x .
\end{aligned}
$$

By assumption, $-h^{\alpha-\varepsilon} \geq-h^{1 / \beta+\varepsilon_{0}+2 \varepsilon}$. Hence, Corollary V.6 implies that the right hand side of (12) is not larger than

$$
\begin{aligned}
\frac{2\left(h^{\alpha-\varepsilon}-h^{\alpha+\varepsilon}\right)}{h^{1 / \beta+\varepsilon_{0}}-h^{1 / \beta+\varepsilon_{0}+2 \varepsilon}} e^{\lambda m\left(h^{\alpha-\varepsilon}+h^{1 / \beta+\varepsilon_{0}}\right) \frac{\mu}{\mu-1}} \\
\quad \times \int d Y_{m}(a) \int_{-h^{1 / \beta+\varepsilon_{0}-a}}^{-h^{1 / \beta+\varepsilon_{0}+2 \varepsilon}-a} z(x) d x
\end{aligned}
$$


and $k_{l}$ does not satisfy (16). This implies that

$$
\leq 2 h^{\alpha-1 / \beta-\varepsilon-\varepsilon_{0}} e^{2 M \frac{\mu}{\mu-1}} P\left(\Delta Z \in\left(-h^{1 / \beta+\varepsilon_{0}},-h^{1 / \beta+\varepsilon_{0}+2 \varepsilon}\right) \mid A_{3}\right) \quad L_{j} \leq L_{k_{l}} L^{\left(j-k_{l}\right)(\beta-1-\varepsilon)}<L_{j}^{\beta-1-\varepsilon}
$$

$$
\leq 2 h^{\alpha-1 / \beta-\varepsilon-\varepsilon_{0}} e^{2 M \frac{\mu}{\mu-1}} .
$$

As in the case $\alpha<1 / \beta$, we obtain following inequality with probability 1 (for sufficiently small $h$ ) using Lemma VI.1 and Borel-Cantelli Lemma:

$$
N_{h}^{\varepsilon}(\alpha) \leq 1+1 / \beta-\alpha+\varepsilon \beta+\varepsilon_{0}
$$

Passing with $\varepsilon$ to 0 we get (in regular case only)

$$
f_{g}(\alpha) \leq 1+1 / \beta-\alpha+\varepsilon_{0}
$$

where $\varepsilon_{0}$ may be chosen arbitrarily small.

2) Lower bound, $\beta \in(1,2)$ : We assume $1<\beta<2$ and choose a small enough $\varepsilon_{0}$.

Proposition VI.2. There exist constants $K_{3}, K_{4}$ and a sequence $m_{i} \rightarrow \infty$ such that

$$
\begin{gathered}
N_{m_{i}} \geq K_{3} M_{m_{j}}, \\
\sum_{j>m_{i}} \frac{N_{j}}{L_{j}} \leq K_{4} \frac{N_{m_{i}}}{L_{m_{i}}}, \\
\limsup _{i \rightarrow \infty} \frac{\log N_{m_{i}}}{m_{i} \log L} \geq \beta-1 .
\end{gathered}
$$

Proof:

Instead of (15) we are going to prove that for every sufficiently small $\varepsilon$ the following condition can be satisfied (together with (13) and (14)):

$$
N_{m_{i}} \geq L^{m_{i}(\beta-1-\varepsilon)} .
$$

Indeed, if (16), (13) and (14) can be all satisfied for arbitrarily small $\varepsilon$ and arbitrarily large $m_{i}(\varepsilon)$ then we may choose a subsequence satisfying (15) as well.

Choose a small $\varepsilon$ and let $K_{3}=1-L^{-\beta+1+\varepsilon_{0}}$. If (13) is satisfied only finitely many times then for all $j$ greater than some $J$

$$
N_{j}<\left(L^{\beta-1-\varepsilon}-1\right) M_{j-1} .
$$

This implies that for all $j>J$

$$
M_{j}<M_{J} L^{(j-J)(\beta-1-\varepsilon)}
$$

which is in contradiction with the definition of $\beta$. Hence, there is a sequence $k_{i}$ satisfying (13).

If (16) is satisfied for only finitely many $k_{i}$ then for every $j$ greater than some $k_{J}$ we have

$$
k_{l} \leq j<k_{l+1}
$$

for all $j>k_{J}$ which is again in contradiction with the definition of $\beta$. Hence, there exists a subsequence $k_{i}$ satisfying both (13) and (16).

By definition of $\beta$, the sequence $a_{k}=N_{k} L_{k}^{-\beta+1-\varepsilon}$ tends to zero. For any $k_{i}$ we can choose $m_{i} \geq k_{i}$ with

$$
a_{m_{i}}=\max _{j \geq k_{i}} a_{j}
$$

We have

$$
\begin{aligned}
\sum_{j>m_{i}} \frac{N_{j}}{L_{j}} & \leq \frac{N_{m_{i}}}{L_{m_{i}}} \sum_{j>m_{i}} L^{-\left(j-m_{i}\right)(2-\beta-\varepsilon)} \\
& =\frac{1}{L^{2-\beta-\varepsilon}-1} \frac{N_{m_{i}}}{L_{m_{i}}}
\end{aligned}
$$

hence $m_{i}$ satisfies (14). We have

$$
\begin{aligned}
M_{m_{i}} \leq & M_{k_{i}}+\sum_{j=k_{i}+1}^{m_{i}} a_{m_{i}} L^{j(\beta-1+\varepsilon)} \\
\leq & \frac{1}{1-L^{1-\beta+\varepsilon}} L^{k_{i}(\beta-1+\varepsilon)} a_{m_{i}}+ \\
& \frac{1}{1-L^{1-\beta-\varepsilon}}\left(L^{m_{i}(\beta-1+\varepsilon)}-L^{k_{i}(\beta-1+\varepsilon)}\right) a_{m_{i}} \\
\leq & \frac{1}{1-L^{1-\beta+\varepsilon}} L^{m_{i}(\beta-1+\varepsilon)} a_{m_{i}} \\
= & \frac{1}{1-L^{1-\beta+\varepsilon}} N_{m_{i}}
\end{aligned}
$$

hence $m_{i}$ satisfies (13). We also have

$$
\begin{aligned}
N_{m_{i}} & \geq N_{k_{i}} L^{\left(m_{i}-k_{i}\right)(\beta-1+\varepsilon)} \\
& \geq L^{m_{i}(\beta-1-\varepsilon)+2\left(m_{i}-k_{i}\right) \varepsilon}
\end{aligned}
$$

hence $m_{i}$ satisfies (16) as well.

Let $m \in\left\{m_{i}\right\}$ be one of the indices satisfying the assertion of Proposition VI.2 and let

$$
h=\frac{1}{L_{m} N_{m}} .
$$

Using (15) and the definition of $\beta$, we may assume

$$
L_{m}^{-\beta-\varepsilon_{0}} \leq h \leq L_{m}^{-\beta+\varepsilon_{0}} .
$$

Our first goal is to estimate the increments of the tail of $Z$. We start by estimating the variance: 


$$
\begin{aligned}
\operatorname{var} T_{L_{m}} & \leq \sum_{L_{j}=L_{m+1}}^{h^{-1}} K_{0} N_{j} \frac{h}{L_{j}}+\sum_{L_{j}>h^{-1}} K_{0} N_{j} \frac{1}{L_{j}^{2}} \\
& \leq \sum_{L_{j}>L_{m}} K_{0} N_{j} \frac{h}{L_{j}} \\
& \leq K_{0} K_{4} L_{m}^{-2}
\end{aligned}
$$

where the last inequality follows from (14). Let $A_{4}$ be the event stating that $T_{L_{m}} \in\left(-\frac{1}{2} L_{m}^{-1}, L_{m}^{-1} \log |\log h|\right)$. We denote $A_{4}^{+}$the event $T_{L_{m}} \geq L_{m}^{-1} \log |\log h|$ and $A_{4}^{-}$the event $T_{L_{m}} \leq-\frac{1}{2} L_{m}^{-1}$. We will use an auxiliary random variable:

$$
W=\sum_{s} T_{L_{m}}(s)=\sum_{\lambda_{j}>L_{m}}\left(Z_{\lambda_{j}}(t+1)-Z_{\lambda_{j}}(t)\right) .
$$

We have $E W=0$ and

$$
\operatorname{var} W \approx \sum_{L_{j}>L_{m}} N_{j} \frac{1}{L_{j}^{2}} \leq c\left(\varepsilon_{0}\right) L_{m}^{\beta-3+\varepsilon_{0}} .
$$

By Chebyshev inequality,

$$
P\left(|W|<\frac{1}{6} h^{-1} L_{m}^{-1}\right) \geq 1-c\left(\varepsilon_{0}\right) L_{m}^{-\beta-1+3 \varepsilon_{0}} .
$$

We can write $W$ in a different way:

$$
W=\sum_{s ; A_{4}(s)} T_{L_{m}}(s)+\sum_{s ; A_{4}^{-}(s)} T_{L_{m}}(s)+\sum_{s ; A_{4}^{+}(s)} T_{L_{m}}(s) .
$$

We will estimate the third sum by means of the following version of Markov inequality:

Lemma VI.3. Let $B$ be a random variable with expectation 0 and variance $V$. Then for all $x>0$

$$
P(B>x) \leq \frac{V}{x^{2}}
$$

and

$$
P(B>x) \cdot E(B \mid B>x) \leq \frac{V}{x} .
$$

Applying this lemma with $B=T_{L_{m}}$ and $x=$ $L_{m}^{-1} \log |\log h|$ and invoking Lemma VI.1 we get

$$
P\left(\sharp\left\{s ; A_{4}^{+}(s)\right\} \leq \frac{1}{4} h^{-1}\right) \geq 1-16 K_{4}(\log |\log h|)^{-2}
$$

and

$P\left(\sum_{s ; A_{4}^{+}(s)} T_{L_{m}}(s) \leq \frac{1}{6} h^{-1} L_{m}^{-1}\right) \geq 1-6 K_{4}(\log |\log h|)^{-1}$
We assume that (21), (22) and (23) all hold. Hence

$$
h^{-1}-\sharp\left\{s ; A_{4}^{+}(s)\right\} \geq \frac{3}{4} h^{-1}
$$

and

$$
W-\sum_{s ; A_{4}^{+}(s)} T_{L_{m}}(s) \geq-\frac{1}{3} h^{-1} L_{m}^{-1} .
$$

We have

$$
\begin{gathered}
W-\sum_{s ; A_{4}^{+}(s)} T_{L_{m}}(s)=\sum_{s ; A_{4}(s)} T_{L_{m}}(s)+\sum_{s ; A_{4}^{-}(s)} T_{L_{m}}(s) \\
\leq-\left(h^{-1}-\sharp\left\{s ; A_{4}^{+}(s)\right\}\right) \cdot \frac{1}{2} L_{m}^{-1}+ \\
\sharp\left\{s ; A_{4}(s)\right\}\left(\frac{1}{2} L_{m}^{-1}+L_{m}^{-1} \log |\log h| .\right.
\end{gathered}
$$

Substituting (24) and (25) we get

$$
\begin{aligned}
\sharp\left\{s ; A_{4}(s)\right\} & \geq h^{-1} \frac{1}{12+24 \log |\log h|} \\
& \geq h^{-1+\varepsilon_{0}} .
\end{aligned}
$$

We choose a family of $h^{2 \varepsilon_{0}} L_{m}$ disjoint intervals $J_{k}=\left(b_{k}^{-}, b_{k}^{+}\right) \subset(t, t+1)$, each of length $h^{\varepsilon_{0}} L_{m}^{-1}$ and in distance at least $h^{-\varepsilon_{0}} L_{m}^{-1}$ from each other. We can choose it in such a way that there exist $h^{-1+4 \varepsilon_{0}}$ intervals $I_{h}^{s} \subset \bigcup J_{k}$ with $A_{4}(s)$ satisfied.

The idea of the rest of the proof is as follows. For every interval $I_{h}^{s} \subset \bigcup J_{k}$ for which $A_{4}(s)$ is satisfied, we check whether the events $A_{5}(s)$ and $A_{6}(s)$ (defined below) are satisfied. While $A_{5}(s)$ doesn't depend on the past, $A_{6}(s)$ does. However, we may obtain a uniform lower bound for the probability of $A_{6}(s)$ independent of the past. This allows to estimate the number of intervals $I_{h}^{s} \subset \bigcup J_{k}$ for which $A_{4} \cap A_{5} \cap A_{6}$ is satisfied using the strong law of large numbers. Moreover, classical large deviations arguments allow to get almost sure results. We now make this precise.

We need then to estimate from below $P(\Delta Z \in$ $\left.\left(-h^{\alpha-\varepsilon},-h^{\alpha+\varepsilon}\right) \mid A_{4} \cap A_{5} \cap A_{6}\right)$. This probability does in general depend on the past because the distribution of $\Delta Z_{j}$ for some $j$ will not be in stationary state anymore. However, we are able to give some estimations on this distribution and thus give a uniform lower bound for $P\left(\Delta Z \in\left(-h^{\alpha-\varepsilon},-h^{\alpha+\varepsilon}\right) \mid A_{4} \cap A_{5} \cap A_{6}\right)$. This allows us to obtain the lower bound for $N_{h}^{\varepsilon}(\alpha)$ using the strong law of large numbers.

We define the event $A_{5}=\{\omega$ : . for all $j$ such that $\left.\lambda_{j} \leq L_{m-1}, \Delta Z_{j}=h\right\}$. This event depends only on $Z_{j}$ for $\lambda_{j} \leq L_{m-1}$ and is thus independent from $A_{4}$. We can estimate 


$$
\begin{aligned}
P\left(A_{5}\right) & \geq \prod_{\lambda_{j} \leq L_{m-1}} e^{-h \lambda_{j}} \\
& \approx \exp \left(-\sum_{L_{j}<L_{m}} h N_{j} L_{j}\right) \\
& \geq e^{1-K_{3}^{-1}}
\end{aligned}
$$

where the last inequality follows from (13).

The event $A_{6}(s)$ is defined by two conditions. First, of all $\lambda_{j} \in\left(L_{m-1}, L_{m}\right)$, there must be precisely one for which $Z_{j}(s)$ has exactly one jump in $I_{h}^{s}$ (this $j$ will be denoted $M(s)$ ). The second condition is that $Z_{M(s)}$ had no jumps before inside the same $J_{k}$, i.e. $\Delta Z_{M(s)}(S)=h$ for all $I_{h}^{S}, S<s$ contained in $J_{k}$.

As $A_{6}$ depends only on $\lambda_{j} \in\left(L_{m-1}, L_{m}\right)$, it is independent from $A_{4}$ and $A_{5}$. To estimate its probability, note that there are $N_{m} \lambda_{j} \in\left(L_{m-1}, L_{m}\right)$ while the probability of any given one of them having a jump inside $J_{k}$ before is at most

$$
1-e^{-\left|J_{k}\right| L_{m}} \approx\left|J_{k}\right| L_{m}=h^{\varepsilon_{0}} N_{m}^{-1} .
$$

We can thus assume that at least half of them had no jump before. Let $E_{M}^{m}$ denote the event $\left\{\exists \lambda_{M} \in\right.$ $\left.\left(L_{m-1}, L_{m}\right): \Delta Z_{M(s)}(s)<h\right\}$. Then

$$
\begin{array}{r}
P\left(E_{M}^{m} \cap \Delta Z_{M(s)}(S)=h \forall S<s, I_{h}^{S} \subset J_{k}\right) \\
\geq 1-e^{-h L_{m} \cdot \frac{1}{2} N_{m}}=1-e^{-1 / 2}
\end{array}
$$

while

$$
\begin{gathered}
P\left(\forall \lambda_{j} \in\left(L_{m-1}, L_{m}\right), j \neq M, \Delta Z_{j}=h\right) \\
\geq \prod_{L_{m-1}<\lambda_{j} \leq L_{m}} e^{-h \lambda_{j}} \geq e^{-h N_{m} L_{m}}=e^{-1} .
\end{gathered}
$$

We can now apply the law of large numbers to the estimations above. This yields that there are at least $h^{-1+4 \varepsilon_{0}}$ intervals $I_{h}^{s} \subset \bigcup J_{k}$ for which $A_{4}, A_{5}$ and $A_{6}$ are all satisfied. For all those intervals we have

$$
\begin{aligned}
Y_{M(s)}(s) & \geq h \cdot\left(\sharp\left\{\lambda_{j}<L_{m}\right\}-1\right)-\frac{1}{2} L_{m}^{-1} \\
& \geq h N_{m}-\frac{1}{2} L_{m}^{-1}=\frac{1}{2} L_{m}^{-1}
\end{aligned}
$$

and

$$
\begin{aligned}
& Y_{M(s)}(s) \leq h \cdot\left(\sharp\left\{\lambda_{j}<L_{m}\right\}-1\right)+L_{m}^{-1} \log |\log h| \\
& \leq K_{3} h N_{m}+L_{m}^{-1} \log |\log h| \leq\left(K_{3}+1\right) L_{m}^{-1} \log |\log h| .
\end{aligned}
$$

By (18), for sufficiently small $\varepsilon_{0}$

$$
L_{m}^{-1} \geq 2 h^{\alpha+\varepsilon}
$$

Let us sum up what we have arrived at so far. We have found a number of intervals $I_{h}^{s} \subset[t, t+1]$ where the event $A_{4}$ occurs, i.e. we have an estimation on the increments of the tail of $Z$. We choose some larger intervals $J_{k}$ that cover many of them. We then require $A_{5}$ and $A_{6}$ to occur. Taken together, they mean that we know the increments of all $Z_{j}$ except one, denoted $Z_{M(s)}$, for which it is known that it has precisely one jump in $I_{h}^{s}$. However, it is important to realize that, although the choice of $M(s)$ does not depend on $Z_{M(s)}(t+s h), Z_{M(s)}$ is not in the stationary state at time $t+s h$. It is nevertheless possible to estimate the distribution of $Z_{M(s)}(t+s h)$. First, as $Z_{M(s)}$ has no jumps inside $J_{k}$, we have

$$
Z_{M(s)}(t+s h)=t+s h-b_{k}^{-}+Z_{M(s)}\left(b_{k}^{-}\right) .
$$

Second, there are no conditions on the behaviour of $Z_{M(s)}$ between $b_{k-1}^{+}$and $b_{k}^{-}$. As the distance between these points is at least $h^{-\varepsilon_{0}} \lambda_{M(s)}$, by Proposition V.1, we may freely assume that $Z_{M(s)}\left(b_{k}^{-}\right)$is arbitrarily close to the stationary state for $h$ small enough. Denoting by $\tilde{Z}_{M}$ the density of the distribution of the jump $\Delta Z_{M(s)}^{(s)}$ over the interval $I_{h}^{s}$, and using the fact that $Z_{M(s)}$ has precisely one jump inside $I_{h}^{s}$, we get:

$\tilde{Z}_{M}(x)=\frac{1}{h} \int_{0}^{h} g_{M(s)}\left(-(x-h) \frac{\mu}{\mu-1}-y-\left(t+s h-b_{k}^{-}\right)\right) d y$,

where $0 \leq t+s h-b_{k}^{-}<h^{\varepsilon_{0}} L_{m}^{-1}$. By (26) and (27), we may thus write:

$P\left(\Delta Z(s) \in\left(-h^{\alpha-\varepsilon},-h^{\alpha+\varepsilon}\right) \mid A_{4}(s) \cap A_{5}(s) \cap A_{6}(s)\right)$
$\geq \inf _{a} \int_{-h^{\alpha-\varepsilon}-a}^{-h^{\alpha+\varepsilon}-a} \tilde{Z}_{M}(x) d x \geq \inf _{a} \int_{-2 h^{\alpha+\varepsilon}-a}^{-h^{\alpha+\varepsilon}-a} \tilde{Z}_{M}(x) d x$

where the infimum on $a$ is taken in the interval $\left[\frac{L_{m}^{-1}}{2},\left(K_{3}+1\right) L_{m}^{-1} \log (|\log (h)|)\right]$. The argument of $\tilde{Z}_{M}$ in the right hand side of (29) is between $-2 h^{\alpha+\varepsilon}-$ $\left(K_{3}+1\right) L_{m}^{-1} \log (|\log (h)|) \sim-c L_{m}^{-1} \log (|\log (h)|)$ and $-h^{\alpha+\varepsilon}-\frac{L_{m}^{-1}}{2} \sim-c L_{m}^{-1}$. By (28), the values of $\tilde{Z}_{M}$ in this range are of the order of the values of $g_{M(s)}$ in the range $\left(c L_{m}^{-1}, c L_{m}^{-1} \log (|\log (h)|)\right)$, which, by Lemma V.2, is bounded from below by $c L_{m}|\log (h)|^{-c}$. Hence:

$$
\begin{gathered}
P\left(\Delta Z(s) \in\left(-h^{-\alpha-\varepsilon},-h^{-\alpha+\varepsilon}\right) \mid A_{4}(s) \cap A_{5}(s) \cap A_{6}(s)\right) \\
\geq c h^{\alpha+\varepsilon} L_{m}|\log (h)|^{-c} \geq c h^{\alpha+\varepsilon-\frac{1}{\beta-\varepsilon_{0}}}|\log (h)|^{-c} .
\end{gathered}
$$

By the law of large numbers,

$$
N_{h}^{\varepsilon}(\alpha) \geq c h^{-1+4 \varepsilon_{0}+\alpha+\varepsilon-\frac{1}{\beta-\varepsilon_{0}}}|\log (h)|^{-c},
$$




$$
\text { and } f_{g}(\alpha) \geq 1-\alpha+\frac{1}{\beta} \text {. }
$$

3) Lower bound, $\beta=1$ : The proof in this case goes along the same lines as in the case $\beta \in(1,2)$, hence we are not going to write all the arguments. We will note the differences of the two instead.

The major difficulty here is that property (13) cannot be true for $\beta=1$ (think e.g. of the purely exponential case $N_{j} \equiv 1$ ). We shall replace it by (31) below.

Proposition VI.4. There exist constants $K_{3}, K_{4}$ and a sequence $m_{i} \rightarrow \infty$ such that

$$
\begin{gathered}
N_{m_{i}} \geq \frac{K_{3}}{m_{i}} M_{m_{j}}, \\
\sum_{j>m_{i}} \frac{N_{j}}{L_{j}} \leq K_{4} \frac{N_{m_{i}}}{L_{m_{i}}} .
\end{gathered}
$$

Proof:

Let $K_{3}<1$ and denote by $\nu_{j}$ the arithmetic mean of $N_{1}, \ldots, N_{j}$. If (31) is satisfied only finitely many times then for all $j$ greater than some $J$

$$
N_{j}<K_{3} \nu_{j-1},
$$

hence $\nu_{j}<\nu_{j-1}$, i.e. $\nu_{j}$ is decreasing for $j>J$.

$$
N_{j}<K_{3} Z_{J}
$$

for all $j>J$, hence

$$
\nu_{2 J}<Z_{J} \frac{1+K_{3}}{2}, \quad \nu_{2^{n} J}<Z_{J}\left(\frac{1+K_{3}}{2}\right)^{n} .
$$

In effect, $\nu_{j}$ decreases to zero polynomially fast. In particular, it must become smaller than $K_{3}^{-1}$ at some time, and from this moment on $N_{j} \equiv 0$. As this is a contradiction with the definition of our process, there must be a sequence $k_{i}$ satisfying (31).

We know that the sequence $a_{k}=N_{k} L_{k}^{-\varepsilon}$ converges to zero for any positive $\varepsilon$ (from the definition of $\beta$ ). For any $k_{i}$ we can choose $m_{i} \geq k_{i}$ such that $a_{m_{i}}=$ $\max _{j \geq k_{i}} a_{j}$. (32) is satisfied for the same reason as (14) in Proposition VI.2 is satisfied. As for (31),

$$
\begin{aligned}
M_{m_{i}} & \leq M_{k_{i}}+\sum_{j=k_{i}+1}^{m_{i}} a_{m_{i}} L^{j \varepsilon} \\
& \leq \frac{k_{i}}{K_{3}} N_{k_{i}}+\left(m_{i}-k_{i}\right) N_{m_{i}} \leq \frac{m_{i}}{K_{3}} N_{m_{i}} .
\end{aligned}
$$

Let $m \in\left\{m_{i}\right\}$ be one of indices satisfying the assertion of Proposition VI.4 and let

$$
h=\frac{1}{m L_{m} N_{m}} .
$$

We have

$$
L_{m}^{-1-\varepsilon_{0}} \leq h \leq L_{m}^{-1},
$$

hence $|\log h| \approx m$. The variance estimation becomes

$$
\operatorname{var} T_{L_{m}} \leq \sum_{L_{j}>L_{m}} K_{0} N_{j} \frac{h}{L_{j}} \leq K_{0} K_{4} L_{m}^{-2} m^{-1} .
$$

We define the events $A_{4}$ as $T_{L_{m}} \in$ $\left(-L_{m}^{-1} / 2 m, L_{m}^{-1} \log |\log h|\right), \quad A_{4}^{+} \quad$ as $\quad T_{L_{m}} \geq$ $L_{m}^{-1} \log |\log h|$ and $A_{4}^{-}$as $T_{L_{m}} \leq-L_{m}^{-1} / 2 m$. Applying Lemmas VI.3 and VI.1 we get

$$
P\left(|W|<\frac{1}{6 m} h^{-1} L_{m}^{-1}\right) \geq 1-c\left(\varepsilon_{0}\right) L_{m}^{-2},
$$

$P\left(\sharp\left\{s ; A_{4}^{+}(s)\right\} \leq \frac{1}{4} h^{-1}\right) \geq 1-16 K_{0} K_{4} m^{-1}(\log |\log h|)^{-2}$,

$P\left(\sum_{s ; A_{4}^{+}(s)} T_{L_{m}}(s) \leq \frac{1}{6 m} h^{-1} L_{m}^{-1}\right) \geq 1-6 K_{0} K_{4}(\log |\log h|)^{-1}$.

Assuming that all those hold we get

$$
\sharp\left\{s ; A_{4}(s)\right\} \geq h^{-1} \frac{1}{12+24|\log h| \log |\log h|} \geq h^{-1+\varepsilon_{0}} .
$$

We choose the family $J_{k}$, events $A_{5}$ and $A_{6}$ in the same way as before. We obtain

$$
\begin{aligned}
P\left(A_{5}\right) \approx \exp \left(-\sum_{L_{j}<L_{m}} h N_{j} L_{j}\right) & \geq e^{K_{3}^{-1}} \\
P\left(E_{M}^{m} \cap \Delta Z_{M}(S)=h \forall S<s, I_{h}^{S} \subset J_{k}\right) & \geq 1-e^{-h L_{m} \cdot \frac{1}{2} N_{m}} \\
& \approx \frac{1}{2 m} .
\end{aligned}
$$

and

$P\left(\forall_{\lambda_{j} \in\left(L_{m-1}, L_{m}\right), j \neq M} \Delta Z_{j}=h\right) \geq e^{-h N_{m} L_{m}}=e^{-1 / m}$.

Hence there are approximately $h^{-1+5 \varepsilon_{0}}$ (or more) intervals $I_{h}^{s} \subset \bigcup J_{k}$ for which $A_{4}, A_{5}$ and $A_{6}$ are all satisfied. For all those intervals we have

$$
\begin{aligned}
Y_{M(s)}(s) & \geq h \cdot \sharp\left\{\lambda_{j}<L_{m}\right\}-\frac{1}{2 m} L_{m}^{-1} \\
& \geq h N_{m}-\frac{1}{2 m} L_{m}^{-1}=\frac{1}{2 m} L_{m}^{-1}
\end{aligned}
$$

and

$$
\begin{aligned}
Y_{M(s)}(s) & \leq h \cdot \sharp\left\{\lambda_{j}<L_{m}\right\}+L_{m}^{-1} \log |\log h| \\
& \leq K_{3} h m N_{m}+L_{m}^{-1} \log |\log h| \\
& \leq\left(K_{3}+1\right) L_{m}^{-1} \log |\log h| .
\end{aligned}
$$


Using Lemma V.2 and V.3, we get the following lower bound for $P(\Delta Z(s) \quad \in$ $\left.\left(-h^{\alpha-\varepsilon},-h^{\alpha+\varepsilon}\right) \mid A_{4}(s) \cap A_{5}(s) \cap A_{6}(s)\right)$ :

$$
\begin{gathered}
P\left(\Delta Z(s) \in\left(-h^{\alpha-\varepsilon},-h^{\alpha+\varepsilon}\right) \mid A_{4}(s) \cap A_{5}(s) \cap A_{6}(s)\right) \\
\geq h^{\alpha+\varepsilon} L_{m} \min \left(c|\log (h)|^{-c}, c e^{-c(\log (m))^{2}}\right) .
\end{gathered}
$$

The second term is dominant and it decreases slower that any power of $h$. Hence, by the strong law of large numbers,

$$
N_{h}^{\alpha} \geq c h^{-2+6 \varepsilon_{0}+\alpha+\varepsilon}
$$

and

$$
f_{g}(\alpha) \geq 2-\alpha
$$

\section{Conclusion of the proof}

Let us recapitulate the results from the previous sections. In case $\alpha<1 / \beta$ we have proven the upper bound for $N_{h}^{\alpha}$ for all sufficiently small $h$ and the lower bound for all sufficiently small $h$ satisfying certain conditions. This gives the value of large deviation spectrum. Moreover, it is worthwhile noting that, in the regular case, this condition is satisfied for almost all $h$ hence the spectrum is obtained as a limit (rather than as an upper limit).

In the case $\alpha \geq 1 / \beta$, we have proven the upper bound for $N_{h}^{\alpha}$ for all sufficiently small $h$ satisfying certain conditions. Once again, this condition is satisfied for almost all $h$ in the regular case. However, we can only prove the lower bound for $N_{h}^{\alpha}$ if $\beta<2$ and only for certain sequences of $h$. Hence, if $\beta<2$, in the regular case we have obtained the value of the spectrum and in the general case we have a lower bound but we cannot prove that the spectrum is obtained as a limit.

Checking the proofs one notices that we use only certain properties of the sequence $N_{j}$. In particular, we are not interested in $N_{j}$ for $j>>|\log h|$ (we estimate them out by calculating the variance of the tail) and we are not interested in $N_{j}$ for $j<<|\log h|$ either (there are very few $\lambda_{j}$ in this region and we may assume that the corresponding processes $Z_{j}$ are not going to jump). In other words, all the proofs above would work just as well if we assumed only $N_{j} \approx L_{j}^{\beta-1}$ for all $j \approx|\log h|$, whatever the behavior of $N_{j}$ is outside this range.

Consider now a sequence $\left\{N_{j}\right\}$ which has large regions of $j$ for which $N_{j} \approx L_{j}^{\gamma-1}$ with $\gamma$ varying in some range $\left(\beta_{1}, \beta_{2}\right)$. Choosing $h$ in such a region, we may estimate $N_{h}^{\alpha} \approx h^{-f_{\gamma}(\alpha)}$, where $f_{\gamma}$ is the spectrum of regular process with $\beta=\gamma$. Assuming there are infinitely many regions for every $\gamma=k / 2^{l} \in\left(\beta_{1}, \beta_{2}\right)$, the spectrum of the resulting process is thus:

$$
f_{g}(\alpha)=\sup _{\gamma \in\left(\beta_{1}, \beta_{2}\right)} f_{\gamma}=\min \left(\alpha \beta_{2}, 1,1-\alpha+1 / \beta_{1}\right) .
$$

This finishes the proof of the theorem.

\section{REFERENCES}

[1] F. Baccelli and D. Hong, AIMD, Fairness and Fractal Scaling of TCP Traffic, INFOCOM'02, June 2002.

[2] F. Baccelli and D. Hong, Interaction of TCP Flows as Billiards, IEEE/ACM Trans. Networking 13 (4), 841-853, 2005.

[3] J. Barral and J. Lévy Véhel, Multifractal analysis of a class of additive processes with correlated non-stationary increments, Elec. J. Proba. 9, Paper no. 16, 508-543, 2004.

[4] O.L.V. Costa and F. Dufour, Stability and Ergodicity of Piecewise Deterministic Markov Processes, SIAM J. Ctrl Optim., 47 (2), 1053-1077, 2008.

[5] M. Crovella and A. Bestavros, Self-similarity in World Wide Web traffic: Evidence and possible causes, IEEE/ACM Trans. Networking, 6, 835-846, 1997.

[6] T. D. Dang, S. Molnar and I. Maricza, Queuing Performance Estimation for General Multifractal Traffic, Int. J. Comm. Systems, 16 (2), 117-136, 2003.

[7] H. Doi, T. Matsuda and M. Yamamoto, Influences of TCP congestion control mechanisms to multi-fractal nature of generated traffic, IEEE GLOBECOM 2003, 7, 3658-3662, 2003.

[8] H. Doi, T. Matsuda and M. Yamamoto, Performance evaluation of multi-fractal nature of TCP traffic with RED gateway, IEEE Int. Conf. on Local Computer Networks, 400-401, 2004

[9] M.H.A. Davis, Markov Models \& Optimization, Chapman \& Hall/CRC Monographs on Statistics \& Applied Probability, 1993.

[10] D. Down, S.P. Meyn and R. Tweedie, Exponential and Uniform Ergodicity of Markov Processes, Ann. Probab., 23, 1671-1691, 1995.

[11] R. Ellis, Large Deviations for a general Class of Random Vectors, Ann. Prob., 12, 1-12, 1984.

[12] A.Erramilli, O.Nrayan, A.Neidhardt and I.Saniee, Performance Impacts of Multi-Scaling in Wide Area TCP/IP Traffic, Proc. IEEE INFOCOM 2000, 352-359, 2000

[13] R. Gaigalas and I. Kaj, Convergence of scaled renewal processes and a packet arrival model, Bernoulli, 9(4), 671-703, 2003.

[14] C. Houdré and J. Lévy Véhel, Large deviation multifractal spectra of certain stochastic processes, Preprint.

[15] P. Jacquet, Long term dependences and heavy tails in traffic and queues generated by memoryless ON/OFF sources in series, Inria Tech. Rep. RR-3516, 1998.

[16] S. Jaffard, The multifractal nature of Lévy processes, Probab. Theory Rel. Fields, 114, 207-227, 1999.

[17] S. Jaffard, On lacunary wavelet series, Ann. Appl. Probab., 10, 313-329, 2000

[18] W. E. Leland, M. S. Taqqu, W. Willinger and D. V. Wilson, On the self-similar nature of Ethernet traffic , IEEE/ACM Trans. Networking, 2 (1), 1-15, 1994.

[19] J. Lévy Véhel and R. Riedi, Fractional Brownian motion and data traffic modeling: The other end of the spectrum, Fractals in Engineering, J. Lévy Véhel, E. Lutton and C. Tricot, Eds., Springer Verlag, 1997.

[20] J. Lévy Véhel and B. Sikdar, A Multiplicative Multifractal Model for TCP Traffic, Proc. IEEE ISCC, 714-719, 2001. 
[21] J. Lévy Véhel and C. Tricot, On various multifractal spectra, Fractal Geometry and Stochastics III, Progress in Probability, C. Bandt, U. Mosco and M. Zähle (Eds), Birkhäuser Verlag, 57, 23-42, 2004.

[22] B.B. Mandelbrot, Intermittent turbulence in self-similar cascades: divergence of hight moments and dimension of the carrier, J. fluid. Mech., 62, 331-358, 1974.

[23] B.B. Mandelbrot, A class of multinomial multifractal measures with negative (latent) values for the "dimension" $f(\alpha)$, Fractals' Physical Origins and Properties. Proc. Erice Meeting, L. Pietronero, Ed., Plenum Press, New York, 3-29, 1989.

[24] V. Paxson and S. Floyd, Wide area traffic: The failure of Poisson modeling, IEEE/ACM Trans. on Networking, 3 (3), 226-244, 1995.

[25] V. Pipiras, M.S. Taqqu and J.B. Levy, Slow, fast and arbitrary growth conditions for renewal reward processes when the renewals and the rewards are heavy tailed, Bernoulli, 9 (1), 121$163,2003$.

[26] R. H. Riedi and J. Lévy Véhel, TCP Traffic is multifractal: A numerical study, Inria Tech. Rep. RR-3129, 1997.

[27] S. Sarvotham, R. Riedi and R. Baraniuk, Network and user driven alpha-beta on-off source model for network traffic, Computer Networks 48, 335-350, 2005.

[28] W. R. Stevens, TCP/IP illustrated volume 1, Addison Wesley, 1994. 